\title{
Multilane Detection Entropy-Based Fusion Model by using Iterative Seed and Optimized Deep Convolutional Neural Network
}

\author{
Suvarna Shirke, R. Udayakumar
}

\begin{abstract}
In today's world, the conditions of road is drastically improved as compared with past decade. Most of the express highways are made up of cement concrete and equipped with increased lane size. Apparently speed of the vehicle will increase. Therefore there are more chances for accidents. To avoid the accidents in recent days driver assistance systems are designed to detect the various lane. The detected information of lane path is used for controlling the vehicles and giving alerts to drivers. In this paper the entropy based fusion approach is presents for detecting multi-lanes. The Earth Worm-Crow Search Algorithm (EW-CSA) which is based on Deep Convolution Neural Network(DCNN) is utilized for consolidating the outcomes. At first, the deep learning approaches for path location is prepared using an optimization algorithm and EW-CSA, which focus on characterizing every pixel accurately and require post preparing activities to surmise path data. Correspondingly, the region based segmentation approach is utilizing for the multi-lane detection. An entropy based fusion model is used because this method preserved all the information in the image and reduces the noise effects. The performance of proposed model is analyzed in terms of accuracy, sensitivity, and specificity, providing superior results with values $0.991,0.992$, and 0.887 , respectively.
\end{abstract}

Keywords : Segmentation, Multilane Classification, Multilane Detection, Deep Convolution Neural Network(DCNN), Earth Worm-Crow Search Algorithm (EW-CSA), Driver Assistance System (DAS).

\section{INTRODUCTION}

Now a days, most of the analysts are attracted towards the identification of path to yield Intelligent Transportation System (ITS). Because this ITS gives significant data with respect to managing, cautioning, or controlling the practices to drivers so as to make driving more secure and simpler. The primary reason for this system frameworks is to gather significant information for drivers so as to reduce their exertion in safe driving. Drivers need to focus on different conditions, including vehicle speed and direction, the separation between vehicles, passing vehicles, and potential risky or uncommon events ahead.

Revised Manuscript Received on July 22, 2019.

Suvarna Shirke, Research Scholar, Department of Computer Science and Engineering, Bharath Institute of Higher Education and Research, Chennai, India. Assistant Professor at Atharva College of Engineering.

Dr.R.Udayakumar, Professor and Supervisor, Department of Information Technology, Bharath Institute of Higher Education and Research, Chennai, India
The driver assistance system can collect such information a prior, it will greatly reduce the burden of driving for drivers and make driving more secure and simpler. Driving information referenced above could be identified by numerous sorts of devices, for example, a PC vision framework, radar, microwaves, ultrasonic waves and infrared beams, etc. These gadgets can be used to separate different sorts of information from the driving situations. Most of the automobile companies provides advanced driver-assistance systems. These driver assistance systems have been demonstrated to be successful to reduce vehicle accidents and minimizing driver injury risks. Consequently in ongoing time, these driver assistance systems are being quickly embraced in present day vehicles. Independent route is most wanted functionality among the driver assistance capacities offered by driver assistance systems, which in turn is highly dependent to dependable and exact path recognition.

Most of the road accidents are occurs in traffic conditions. These types of accidents occur due to driver's carelessness and tiredness. The Lane Departure Warning (LDW) system by using the cameras to track the lanes is used in most of the applications. This LDW system gives notification to the driver if he is driving outside the lens unintentionally and takes automatic action to avoid wrong lane path. The secure driving tasks is rely upon the vehicle's driver. The position of a vehicle is depends on the road lane markings which helps to LDW for the identification of vehicle. The presentation of path following depends on lighting conditions, path markings quality, and climatic components, similar to day off, and downpour. The plane of the road may perhaps vary in emergence due to neighboring trees, shadows casted by vehicles, tyre markings and construction material used. Therefore it is more chances for wrong classification of getting the information of road markings. It is a challenge to identify the lane markings in the vehicle markings, also the lane marking sometimes completely disappears due to the heavy traffic conditions. It is also difficult to identify the side lanes due to the heavy traffic. Most of the papers presents the detection of ego lane instead of side lane. The traditional computer vision techniques are used for the most of lane detection in which hand-made highlights are planned by difficult procedure offline tuning.

But, this situation is not vigorous enough in complex driving conditions. Therefore in this article designed a computer vision approach with Earth Worm- Crow Search Algorithm (EW-CSA) which is based on Deep Convolution




Neural Network (DCNN) is utilized for consolidating the outcomes for the lane detection.

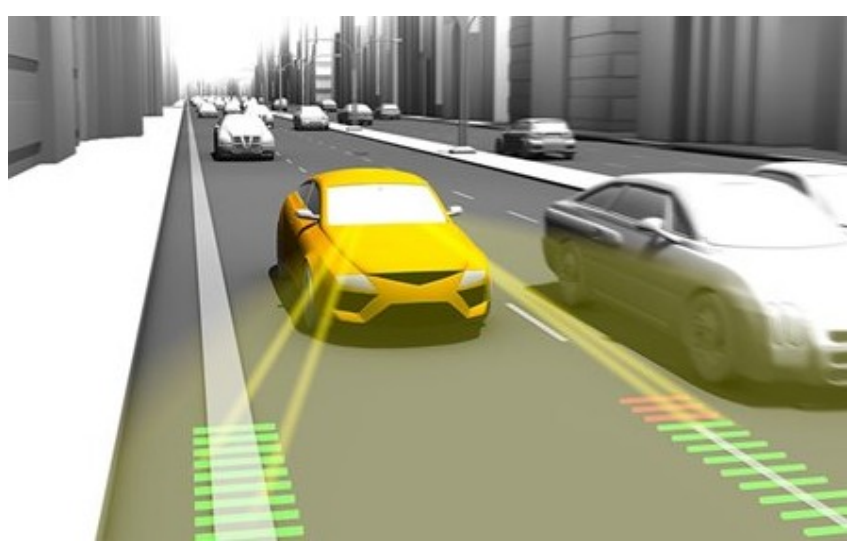

Fig. 1.Example of Lane Detection.

In this proposed method a camera is mounted on the car or vehicle. This camera is used to determine the navigation and paths of the road. The picture obtained from the camera are used for extracting the information of traffic. The information obtained are direction of vehicle, road boundaries and road markings. Most of the images captured by camera are noisy, so it is very difficult to identify the correct lane for the boundary-detection algorithms.

This problem is solved in this paper. A robust method for boundary-detection of lane is present in this paper which can removes the noises presents in the image. Figure 1 shows the example of lane detection. Here, the outputs obtained by region based iterative seed method and EW-CSA based DCNN are integrates with entropy based fusion model for successful multilane recognition. The EW-CSA based DCNN undergoes image transformation using the multiple lane images to obtain Bird's eye view image, and then, the lane detection is done by Earth Worm- Crow Search Algorithm (EW-CSA) based DCNN. The EW-CSA was designed by integrating Earthworm optimization algorithm (EWA) and Crow Search Algorithm (CSA) and is used as the training algorithm in the Deep Convolution Neural Network (DCNN). Then, the second phase undergoes multi-lane detection using region based iterative seed segmentation approach. Entropy is a significant factor that can be utilized for measuring the information content of the images and poses the ability to distinguish the information and noise. Therefore, the information is preserved due to the entropy function which is designed in this proposed system. The results are integrated by both EW-CSA based DCNN and region based segmentation method for effective multi-lane detection.

The association of the paper is: Section 2 gives the literature review of different existing techniques for lane detection. Area 3 talks about the methods used in the proposed system and building block diagram of projected structure. Segment 4 is performance metrics. The outcomes and talks are clarified in area 5 lastly, segment 6 represents the conclusion of paper.

\section{LITERATURE SURVEY}

The review of the existing methods for lane detection is discussed in this section along with the advantages and disadvantages.
Umar Ozgunalp et al. [17] planned a path identification calculation to identify various paths utilizing the example of information pictures. This calculation identifies the path regardless of whether the traffic considered was thick and high. The air-suction pads were mounted on the vehicles and in this manner, had reduce adjustments in roll angle of camera. The path location is completed by assessing the dense vanishing point and utilizing the acquired vanishing points for for path recognition. The technique is increasingly adaptable and the client can process with no outside cameras. In any case, the strategy isn't applicable with spline models and needs advanced methodologies for tracking paths.

Apurba Das et al. [18] planned a binarization calculation by improving the Min-Between-Max Thresholding (MBMT) calculation. The ground truth is utilized as the gauge outline for remedying the little mistakes, which can make extreme modifications in the presentation of the structure. The distinguished consequences of the MMBMT are considered as productive utilizing worldwide MBMT. The technique was utilized for addressing the outlier dismissal and it dealt with the impact of shadow, enlightenment variety in an automated way and tended to the issue of old style time-slice-based based methodology for deciding the ground truth.

Soonhong Jung et al. [19] built up the path identification model utilizing spatiotemporal pictures. This procedure implements the tasks, for example, scaling and binarization utilizing dominant parallel lines for distinguishing the paths. This method requires less time for calculation as compared to other existing strategies and improves the rate of recognition. The Hough change was utilized for binarizing the adjusted spatiotemporal picture to produce parallel straight lines. The technique was strong and eliminates the absent or blocked paths for successful path discovery.

Heba Aly et al. [20] planned a path location model, named LaneQuest, for deciding the paths effectively. The LaneQuest technique distinguishes the paths of street using advanced mobile phones and is said to be energy efficient. The cameras and advanced mobile phones are utilized together to build the path location probability during intense climate conditions. The strategy can deal with the noise of sensors and its ambiguous location. This technique likewise utilized unaided publicly supporting methodology for leaning the position and path span distribution.

Andrade, D.C et al. [21] built up a street path location and tracking technique for identifying the paths and tracks the functional requirement for sending the highlights to DAS, which includes LDW and path keeping assist. Here, the information picture is limited for improving the sharpness and the Regions of interest (ROI) is described by utilizing least safe distance with the vehicles. Consequently, the path tracking technique is adjusted for acquiring the smooth paths. The technique gave improved accuracy levels by utilizing shapes of the roads.

Suddamalla, U et al. [22] structured an efficient calculation to distinguish exact lane inbounds, considering a few road conditions, for example, straight, dashed path markings, and curvy. This technique uses the edges refined from the frames and the data dependent on lane masks for deciding the lane limits.

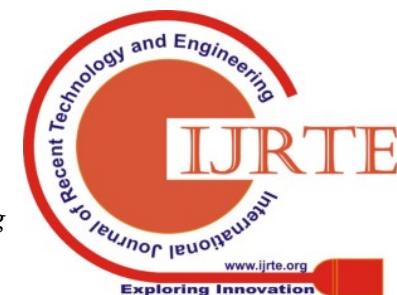


This technique can be used for various real time mechanisms for identifying the paths, yet doesn't think about unintentional edges because of traffic sign and negative climate conditions. The technique utilized shape based outlier rejection, and versatile figure extraction for removing the noise for path detection. This strategy helps to discard unintended edges caused because of traffic signs imprinted on street, in unfavorable climate conditions.

Revilloud, $M$ et al. [23] built up a unconventional methodology for identifying the various paths and tracking is devised on the basis of reactive multi-agent system. This methodology adjusts better record of the particular highlights of street markings in a precise manner. This methodology depends on confidence map and multi-operator model without path marking features. It gave advanced strategies to improve path identification, however it is complex while implementing. The methodology is powerful for deciding the topology of path markings and helps to filter the estimations that can be considered as exceptions or noisy data. However, it neglected to utilize directions for facilitating the propagation of agents in the street markings.

Ali, $\mathrm{M}$ et al. [24] planned a system, named road lane detection system, for recognizing the paths utilizing the dynamic videos that are acquired from the on-board camera mounted on moving vehicles. The vehicle detection operation uses a sober operator and a bounding box detector for detecting the vehicles from the background. Here, the Hough transform was applied for tracking the loosen lane marks by counting the lost frames. This system utilizes the integrated path location, picture subtraction, and vanishing point for path identification. But this method leads to failure if one of the components did not work as each component is linked to each other. In addition, the tracked lanes cut across the vehicle in tracking and thus, masking is essential for processing the image, but information of the vehicle is lost in this process.

\section{PROPOSED ENTROPY BASED ITERATIVE SEED AND OPTIMIZED DCNN FOR MULTI-LANE DETECTION}

This segment outlines the proposed entropy based combination model created for performing the multilane location. Here, two strategies, to be specific EW-CSA based DCNN and region based iterative seed strategy, alongside the entropy function, are utilized for identifying multi-paths. Figure 1 represents the block diagram of the multi-path detection model utilizing proposed fusion model based on entropy measure.

The reason for the multi-path location plan is to decide the paths from multi street path pictures to give safe driving. The proposed model utilizes entropy function for playing out the multilane detection. The fusion approach is devised recently by based EW-CSA based DCNN and the region based segmentation. The fusion approach depends on the entropy measure, which fuses the output created from the first and the second phases for performing multi-path detection.

\section{A. Detection of Road Lane Pictures}

Road lane pictures detection is most complicated work. Most of the researchers either uses the color information or shape information to detect the road lane.



Fig. 2.Block Diagram Of Multi-Lane Detection Model Using Proposed Entropy Fusion Model.

It is hard to catch a great picture for street path detection which contains increasingly number of surface or highlights of the picture. Yet, in this framework we get the street path pictures with adequate sharpness and resolution, Less number of artifacts in infrared light. Additionally we get appropriate light of inside street path design.

\section{B. Pre-processing and De-Noising}

The per-processing stage is utilized to improve the various parameters an lane detection pictures, for example, contrast, intensity, signal to noise ratio, etc.

Additionally it is utilized to expand the nature of a picture because of undesired distortions. This progression upgrades the features of lane detection picture which are required for further handling. It just upgrades the nature of picture, it doesn't expands the data substance of a picture. The intensity range of an lane picture is standardized to [0 1]. This range of intensity values demonstrates the most intensity value by partitioning all intensity values. The diffusion filter process is clarified in [11] is utilized to expand the intensity and contrast of an lane picture. The signal to noise ratio of lane picture is improved with the assistance of anisotropic dispersion filter.

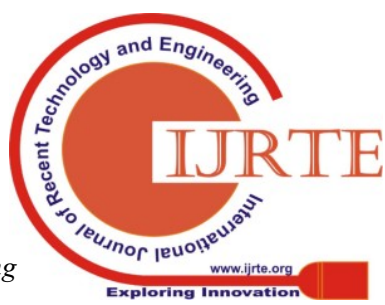


The instrument which is utilized for this reason for existing is MatLab understand the numerous brightness transformations. The noises shot and impulse noise are rejected by the median filter. To find the rank-order information and spatial information of an iris image a weighted median (WM) filter is used and this is one of the type of median filter.

\section{Image Segmentation}

Presently the caught pictures are given to the detection module. The primary work of the detection module is to segment the input caught by picture and extract out the areas and contain road lane patterns. With the help of color segmentation division method unwanted background picture can be removed. The segmentation is the first step of this lane detection system. The main aim of this method is to detect the lane borderline, layout, centers, etc.

\section{Entropy Based Fusion Model for the Multilane Detection}

The entropy based fusion model for the multilane detection have proven to be more robust method. This section reports the proposed entropy function model. This model is designed to perform multilane detection of a path. The source images which contains information of fused images are evaluated by adapting the entropy based approach. Therefore the function for effective multilane detection, the proposed model fuses the output computed by the EW-CSA based DCNN and region based iterative seed method which is based on the entropy function. In the first stage of this work, the output images are observed in two phase. In a first phase it measures the distance of road and in second phase it measures the lane distance.

The multiple lane images are obtained and at the first step, these multiple lane images will be given as the input of the driver assistance system. The multiple lane image will be then transformed into its corresponding Bird's eye view image using the transformation process, which will involve the use of cameras on the vehicle to stitch the views from the cameras. This transformation technique will provide the driver with a clear image of the area behind the car. The bird's eye view image will be the input to the Deep Convolution Neural Network (DCNN) classifier. Figure 3 shows the calculation of distance.

At first, the multilane recognition is done utilizing by EW-CSA based DCNN. Here, the input multi-lane pictures are exposed to the image transformation for preparing and changing the picture from one domain to another domain. The picture transformation is done utilizing inverse perspective mapping for changing over the various lane pictures to the bird's eye view picture.

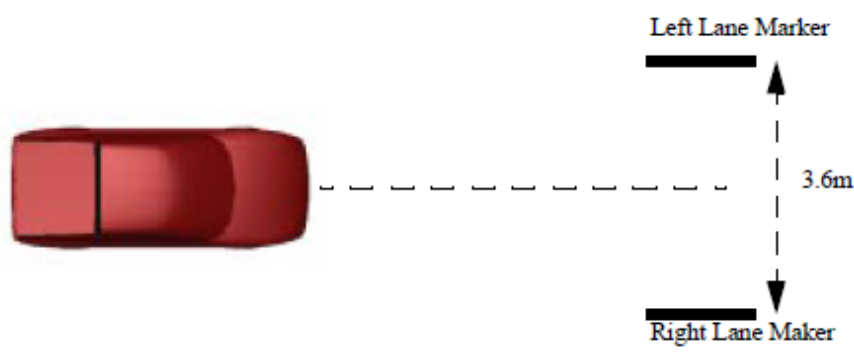

Fig. 3.Calculation of Distance.
When the bird's eye view picture is gotten, it is given as the input to the DCNN classifier for distinguishing the lane paths. At that point, the EW-CSA, which is the mixture of Earthworm optimization algorithm (EWA) and Crow Search Algorithm (CSA), is used for choosing the ideal weights via preparing the DCNN. Coordinating EWA and CSA can tackle the design issues and optimization issues in EW-CSA and can escape from neighborhood minima trap. The DCNN includes number of convolution layers, pooling layers, and Fully Connected layers, in which each layer is utilized to perform a specific task. The initial step for preparing the DCNN is initialization of weights that are resolved by utilizing EW-CSA and the update is performed based on weights that contribute towards minimum error value. The update dependent on EW-CSA is considered for controlling the streamlining procedure. Consequently, the changed Cauchy transformation condition for EW-CSA is detailed as,

$M_{k, l}=\frac{\eta_{k} * H^{k, m}-1}{\eta_{k} * H^{k, m}}\left(K_{l} * L-\frac{\eta_{k} * H^{k, m} * h^{l, m}}{1-\eta_{k} * H^{k, m}}\right)$

where, $\eta_{k}$ signifies arbitrary number in range $[0,1], H_{k, m}$ speaks to flight length, $K_{1}$ shows weight vector, $L$ refers to Cauchy distribution random number, and $\mathrm{h}_{1, \mathrm{~m}}$ determines the memory of best arrangement in $\mathrm{m}^{\text {th }}$ iteration.

In this manner, the weights in DCNN are refreshed dependent on EW-CSA and the weights relating to the minimum value of error are adapted for training DCNN when the algorithm reaches the maximum iteration.

\section{E. Multi-Lane Detection Based On Region Based Iterative Seed Method}

To feed the information presents in a picture, the segmentation method, namely region based iterative seed method, is utilized for the multilane identification, and this method is depicted in this section. For this procedure, at first, the input picture experiences segmentation using the sparking method. The sparking strategy is adapted in the picture to improve the viability of the picture by utilizing an an estimated threshold value. At first, the sample of input picture experiences the sparking procedure for acquiring the segmented picture and after that,

approach, named area based iterative seed, is utilized for recognizing the lanes. In the region based iterative seed approach, the picture is partitioned into matrices, and the targets are chosen in random. After that, the Bhattacharyya distance is calculated between the grids and the targets. the grids that have that have least separation are chosen and they have a place with the specific targets. The Bhattacharyya separation can be used for deciding the general closeness of the two samples and is utilized for estimating the separability of classes and is more dependable than other separation measures. The Bhattacharyya distance is adapted for computing the features in a two pattern recognition problem. The grid points indicates that have belong to the relating targets are averaged to form the target at the next iteration. The technique is repeated until the last

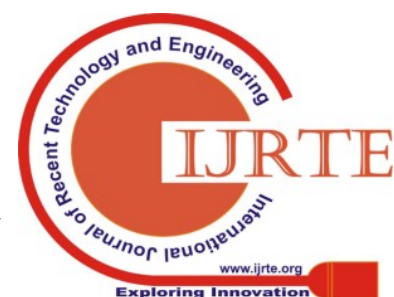


targets are determined, and after that, grouped to separate the lanes from the roads based on the nearest neighbor distance measure. The closest neighbor distance measure is simpler to implement and executes in less time. The closest neighbor distance measure is simpler to implement and executes in less time. This measure gives exact outcomes while evaluating the final targets. Thus, the region-based iterative seed technique identifies the patterns of roads and lanes dependent on maximum occurred cases.

\section{F. Entropy Based Fusion Model For The Multilane Detection}

In this area, the proposed model, created utilizing the entropy work, for playing out the multilane location is explained. The entropy is adapted for assessing the information that fused picture contains about the source pictures. Accordingly, the proposed model fuses the output processed by the EW-CSA based DCNN and region based iterative seed method based on the entropy function for effective multilane identification. From the previous phases, the output is observed in two categories that is either lane or road depending on the nearest neighbor distance measure. The output generated by the first phase and second phase are given as an input to the fusion approach, and the two regions, i.e. roads and lanes. The pixels which are belongs to the lanes are denoted as 1 and the pixels belongs to the road segment are denoted by 2 . Once the pixels of multilane are extracted, the probability of occurrence of the lane pixels from first stage and second stage is determined. These pixels are represents in matrix and denoted as $\mathrm{P}_{1}$. Then, the probability of occurrences dependent on neighborhood pixels is determined independently for the result of first stage and that of second stage and is represented as the matrices. This matrix is denoted as $\mathrm{P}_{2}$ and $\mathrm{P}_{3}$. At last, combination of pictures is done by taking the average of probabilities gained and the average of $\mathrm{P}_{1}, \mathrm{P}_{2}$ and $\mathrm{P}_{3}$. It is denoted as $\mathrm{P}$. Then, the entropy of the obtained probability is computed for fusing the outputs of first and second stage for multilane detection. The pixel wise processing is employed for executing a standard operation on each pixel position for generating an output and then, evaluate the next pixel position and iterate the sample process, till all the pixels are processed.

\section{PerformanCe Metrics}

The metrics employed for the analysis include Specificity, Sensitivity and Detection accuracy and they are formulated as follows,

\section{A. Detection Accuracy}

It refers to the average rate of correct lanes detected by the algorithm against the ground truth information.

$$
\text { Accuracy }=\frac{T p+T n}{T p+T n+F p+F n}
$$

where, Tp, Tn ,Fp and Fn, shows the true positive, true negative, false positive, and false negative, respectively.

\section{B. Sensitivity}

It is a measure to identify correctly detected lanes and is referred as true positive rates.

$$
\text { Sensitivity }=\frac{T p}{T p+F n}
$$

\section{Specificity}

It is a measure to identify the correctly rejected lanes and is also known as False Positive Rate (FPR).

$$
\text { Specificity }=\frac{T n}{T n+F p}
$$

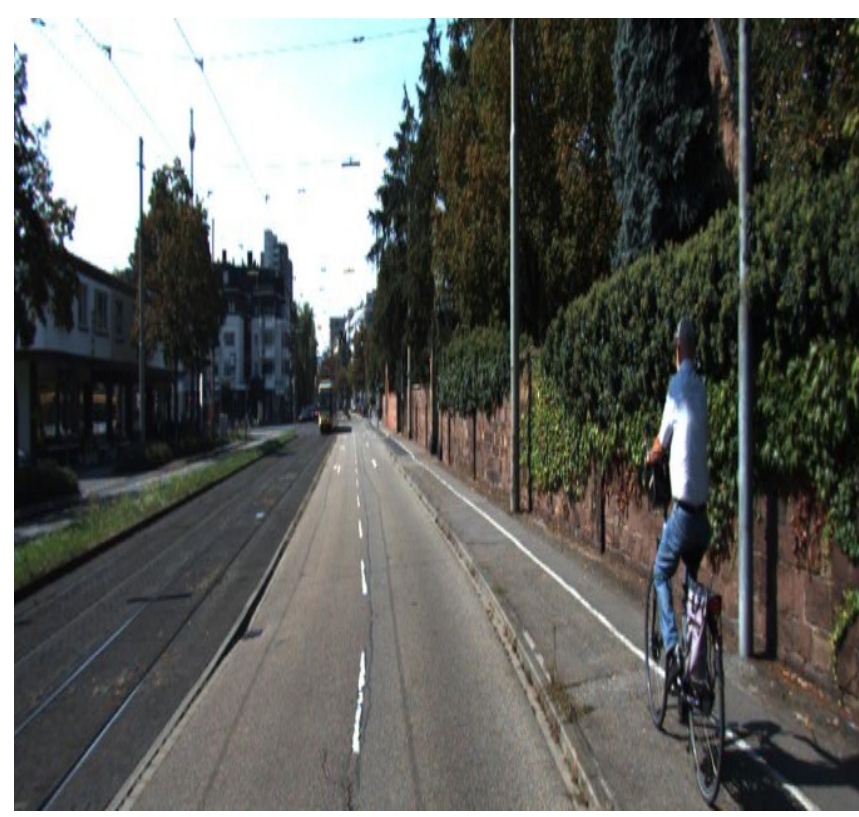

Fig. 4.Captured Picture of Road with Lane.



Fig. 5. Image detected by EW-CSA based DCNN classifier.

\section{EXPERIMENTAL RESULTS}


The step by step execution of the proposed framework is displayed. Here observational results are characterized in this unit of proposed framework.

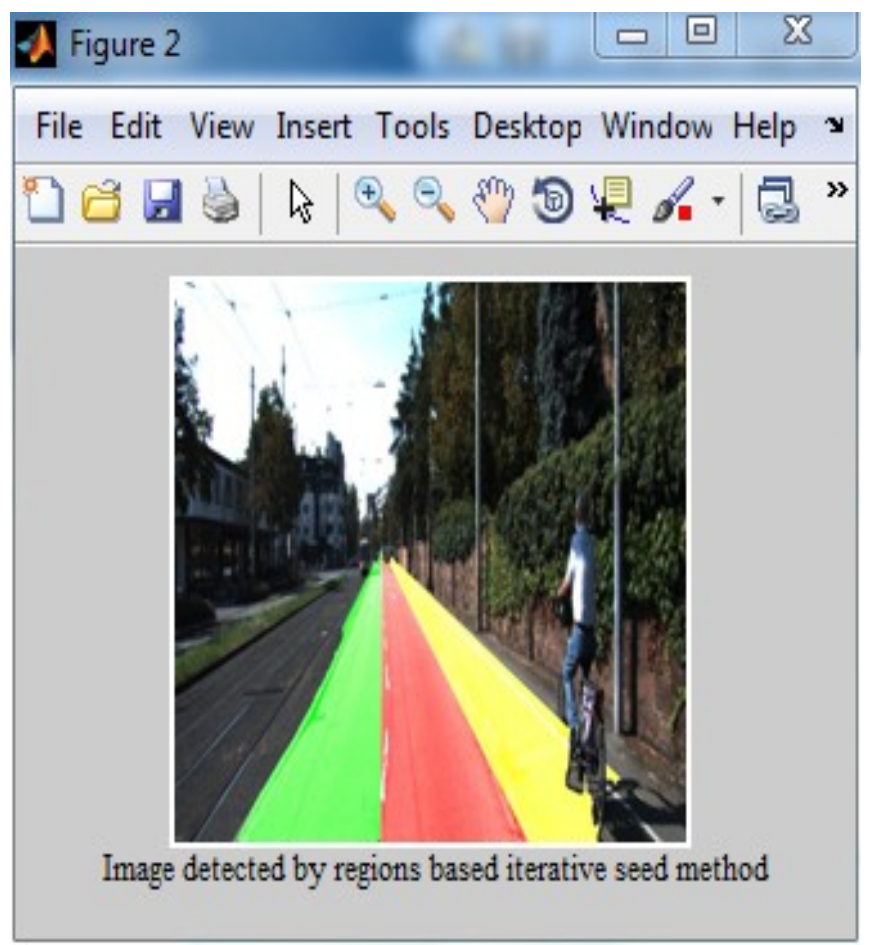

Fig. 6. Image detected by regions based iterative seed method.

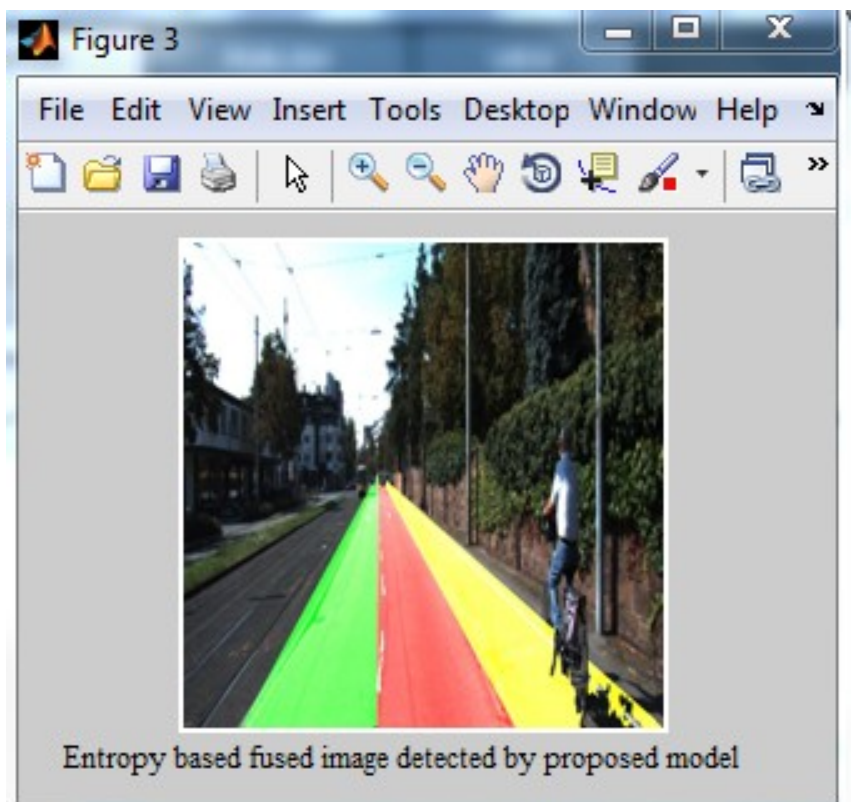

Fig. 7. Entropy Based Fused Image Detected By Proposed Model.

Firstly, take input road image with lane. To get the road scene pictures were taken in by great camera with 14 megapixels, so that there was a decent appropriation of pictures taken under various lighting conditions and at various points. The pictures in every class were randomly chosen. Each video sequence was down sampled to a frame rate of $5 \mathrm{~Hz}$ before being submitted to the system. Test video sequences were acquired from video camera which is mounted in a vehicle were utilized for the contribution to this system. Figure 4 shows the picture captured of road with lane. Figure 5 shows the picture of Image detected by EW-CSA based DCNN classifier. Figure 6 shows the picture of Image detected by regions based iterative seed method. Figure 7 shows the picture of Entropy based fused image detected by proposed model.

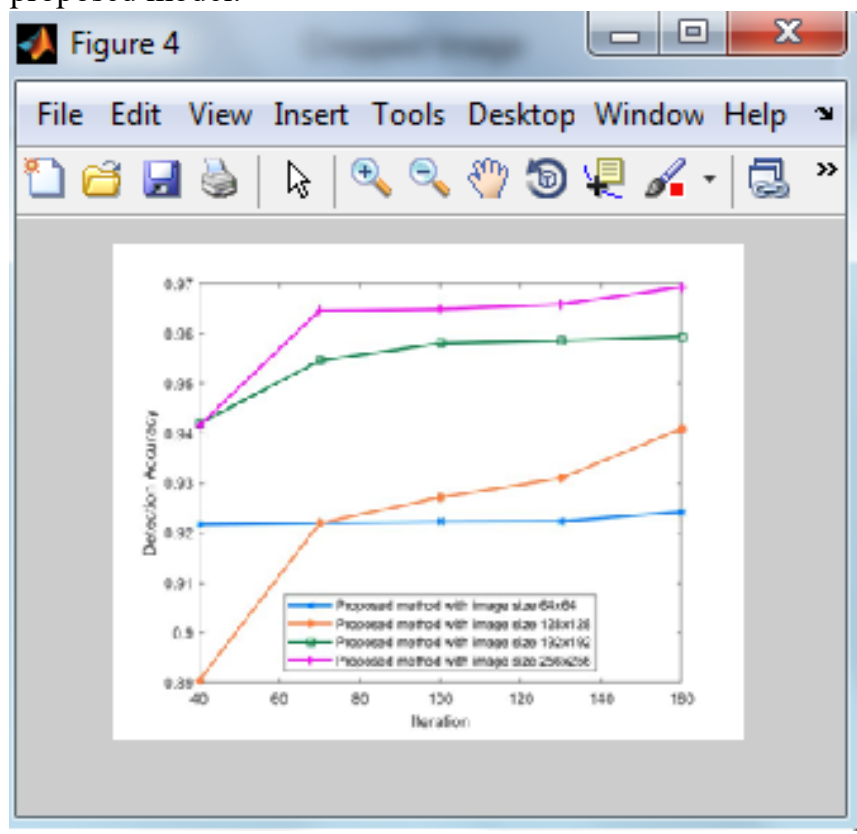

Fig. 8. Captured Picture Accuracy.

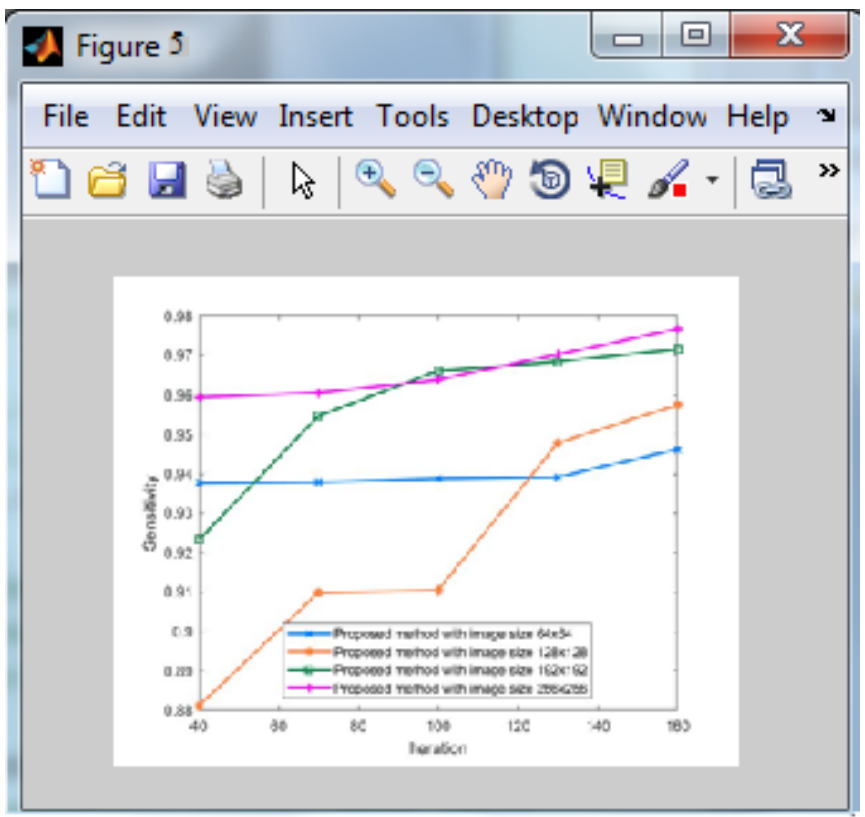

Fig. 9. Captured Picture Sensitivity.

Figure 8 shows the picture of accuracy of captured picture. Figure 9 shows the picture of sensitivity of captured picture. Figure 10 shows the picture of specificity of captured picture

\section{CONCLUSION}

This paper plans a strategy for helping the Driver-assistance system for preventing the accidents. The lane detection is done by utilizing the proposed entropy based fusion model for identifying numerous lane.

Here, the proposed model is used for integrating the results

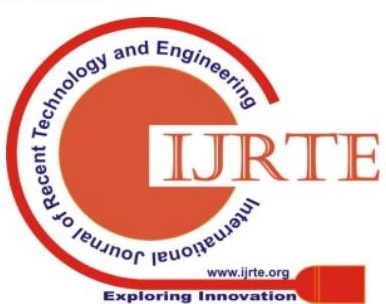


obtained by two phases for multilane detection. In the first phase, the multi-lane detection is performed using EW-CSA based DCNN, wherein EW-CSA is employed to train the DCNN.

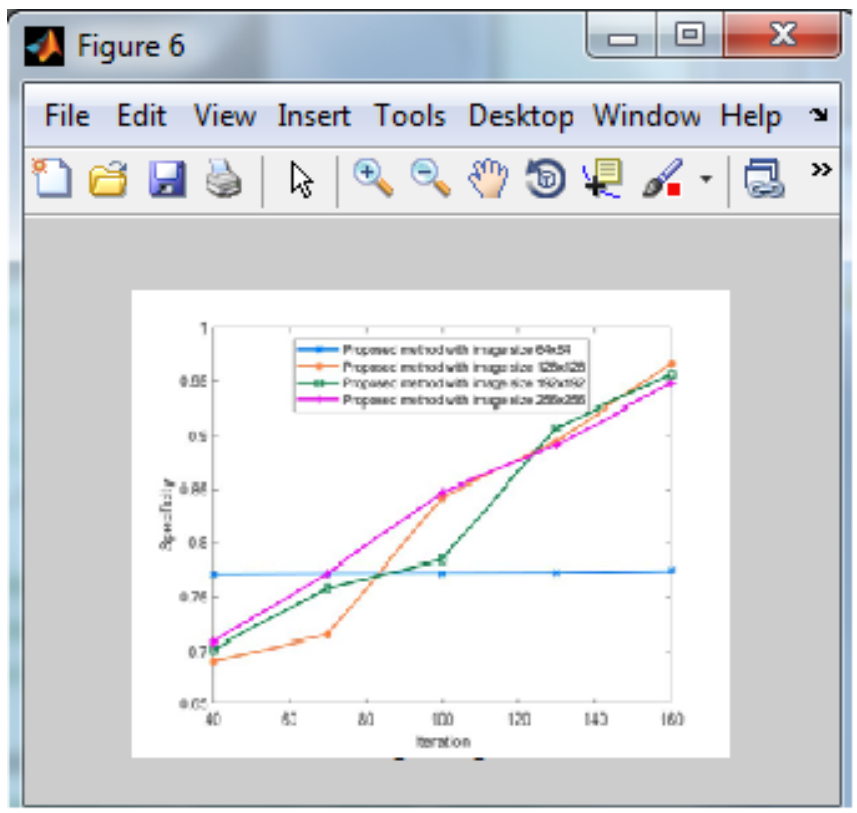

Fig. 10. Captured Picture of Specificity.

Then, the proposed entropy based fusion model is employed to make the final decision by combining the results of both the techniques based on the entropy measure. The performance of proposed model provides superior results in terms of accuracy, sensitivity, and specificity with values $0.99,0.99$ and 0.88 respectively.

\section{REFERENCES}

1. Clanton, J.M., Bevly, D.M. and Hodel, A.S., "A low-cost solution for an integrated multisensor lane departure warning system," IEEE Transactions on Intelligent Transportation Systems, vol.10, no.1,

2. W. Jones, "Building Safer Cars," IEEE Spectrum, vol. 39, no. 1, pp. 82-85, Jan. 2002.

3. Geronimo, D., Lopez, A.M., Sappa, A.D. and Graf, T., "Survey of pedestrian detection for advanced driver assistance systems," IEEE transactions on pattern analysis and machine intelligence, vol.32, no.7, pp.1239-1258, 2010.

4. Kozak, K., Pohl, J., Birk, W., Greenberg, J., Artz, B., Blommer, M., Cathey, L. and Curry, R., "Evaluation of lane departure warnings for drowsy drivers," In Proceedings of the human factors and ergonomics society annual meeting, vol. 50, No. 22, pp. 2400-2404, October, 2006.

5. J.C. McCall and M.M. Trivedi, "Video-based Lane Estimation and Tracking for Driver Assistance: Survey, System, and Evaluation”, IEEE Transactions on Intelligent Transportation Systems, vol.7, pp.20-37, 2006.

6. S.G. Jeong, C.S. Kim, K.S. Yoon, J.N. Lee, J.I. Bae, and M.H. Lee, "Real-time Lane Detection for Autonomous Navigation",In proceedings of international conference on Intelligent Transportation Systems, pp. 508-513, 2001 lateral offset with uncalibrated camera," In proceedings of international conference on Intelligent Transportation Systems, pp. 102-107, 2005.

8. Kaur, G. and Kumar, D., "Lane detection techniques: A review," International Journal of Computer Applications, vol.112, no.10, 2015.

9. Yu, B., Zhang, W. and Cai, Y., "A lane departure warning system based on machine vision," In Computational Intelligence and Industrial Application, vol. 1, pp. 197-201, December 2008.

10. Hillel, A.B., Lerner, R., Levi, D. and Raz, G., "Recent progress in road and lane detection: a survey," Machine vision and applications, vol.25, no.3, pp.727-745, 2014.

11. K. Kluge, S. Lakshmanan, "A deformable template approach to lane detection, in Intelligent Vehicle, vol.25, pp . .54-59, September 1995. pp.47-59, 2009.

7. Jung, C.R. and Kelber, C.R., "A lane departure warning system using

12. D. Jung Kang, J. Won Choi, I.S. Kweon, "Finding and tracking road lanes using line-snakes," Proceedings of Conference on Intelligent Vehicle, pp. 189-194, 1996.

13. A. Kaske, D. Wolf, R. Husson, "Lane boundary detection using statistical criteria," International Conference on Quality by Artificial Vision, pp.28-30, 1996.

14. Wang, Y., Teoh, E.K. and Shen, D., "Lane detection and tracking using B-Snake," Image and Vision computing, vol.22, no.4, pp.269-280, 2004

15. Bertozzi, M., Broggi, A. and Fascioli, A., "Vision-based intelligent vehicles: State of the art and perspectives," Robotics and Autonomous systems, vol.32, no.1, pp.1-16, 2000.

16. Hur, J., Kang, S.N. and Seo, S.W., "Multi-lane detection in urban driving environments using conditional random fields," In Intelligent Vehicles, pp. 1297-1302, June 2013.

17. Umar Ozgunalp, Rui Fan, Xiao Ai, and Naim Dahnoun, "Multiple Lane Detection Algorithm Based on Novel Dense Vanishing Point Estimation", IEEE Transactions On Intelligent Transportation Systems, Vol. 18, No. 3, March 2017.

18. Apurba Das, Member, Siva Srinivasa Murthy, and Upendra Suddamalla, "Enhanced Algorithm of Automated Ground Truth Generation and Validation for Lane Detection System by M2BMT", IEEE Transactions On Intelligent Transportation Systems, Vol. 18, No. 4, April 2017.

19. Soonhong Jung, Junsic Youn, and Sanghoon Sull, "Efficient Lane Detection Based on Spatiotemporal Images", IEEE Transactions On Intelligent Transportation Systems, January 2015.

20. Heba Aly, Anas Basalamah, and Moustafa Youssef, "Robust and ubiquitous smartphone-based lane detection", Pervasive and Mobile Computing, 2015.

21. Andrade, D.C., Bueno, F., Franco, F.R., Silva, R.A., Neme, J.H.Z., Margraf, E., Omoto, W.T., Farinelli, F.A., Tusset, A.M., Okida, S. and Santos, M.M., "A Novel Strategy for Road Lane Detection and Tracking Based on a Vehicle's Forward Monocular Camera," IEEE Transactions on Intelligent Transportation Systems, vol.99, pp.1-11, 2018.

22. Suddamalla, U., Kundu, S., Farkade, S. and Das, A., "A novel algorithm of lane detection addressing varied scenarios of curved and dashed lanemarks," In Image Processing Theory, Tools and Applications, pp. 87-92, November 2015

23. Ali, M., Radzi, A. and Saad, H.M, "A New Approach to Highway Lane Detection using Hough Transform Technique," Journal of ICT, vol.16, no.2, pp.244-260, 2017

24. Gai-Ge Wang, Suash Deb, and Leandro Coelho, "Earthworm optimization algorithm: a bio-inspired metaheuristic algorithm for global optimization problems", International Journal of Bio-Inspired Computation, October 2015

25. A. Askarzadeh, "A novel metaheuristic method for solving constrained engineering optimization problems: Crow search algorithm," Computers \& Structures, vol. 169, pp.1-12, 2016.

26. Alexander Rakhlin, Alexey Shvets, Vladimir Iglovikov, and Alexandr A. Kalinin, "Deep Convolutional Neural Networks for Breast Cancer Histology Image Analysis", International Conference Image Analysis and Recognition ICIAR, Image Analysis and Recognition, pp. 737-744 2018.

27. Mane, V.M. and Jadhav, D.V., "Holoentropy enabled-decision tree for automatic classification of diabetic retinopathy using retinal fundus images," Biomedical Engineering/Biomedizinische Technik, vol.62, no.3, pp.321-332, 2017.

28. Wang, P., Fu, H. and Zhang, K., "A pixel-level entropy-weighted image fusion algorithm based on bidimensional ensemble empirical mode decomposition," International Journal of Distributed Sensor Networks, vol.14, no.12, pp.1550147718818755, 2018.

\section{AUTHORS PROFILE}



Suvarna Shirke-Pansambal is the Research Scholar at Bharath Institute of Higher Education and Research, Department of Computer Science \& Engineering, Chennai. She is working as an Assistant Professor in Atharva College of Engineering, Mumbai. 
Dr.R.Udayakumar is Professor at Department of Information Technology, Bharath Institute of Higher Education and Research, Chennai, India. He has published more than 600 papers and he is Supervisor for Research Scholars at BIHER. 